DESY 98-083

July 1998

\title{
Naturally light sterile neutrinos
}

\author{
UTPAL SARKAR $^{(a, b)}$ 巴 \\ ${ }^{(a)}$ Theory Group, DESY, Notkestraße 85, 22603 Hamburg, Germany \\ ${ }^{(b)}$ Theory Group, Physical Research Laboratory, Ahmedabad, 380 009, India ?
}

\begin{abstract}
A simple model to accomodate light sterile neutrinos naturally with large mixing with the usual neutrinos has been proposed. The standard model gauge group is extended to include an $S U(2)_{S}$ gauge symmetry. Heavy triplet higgs scalars give small masses to the lefthanded neutrinos, while a heavy doublet higgs scalar give mixing with the sterile neutrinos of the same order of magnitude. The neutrino mass matrix thus obtained can explain the solar neutrino deficit, the atmospheric neutrino deficit, the LSND data and hot dark matter. Lepton number is violated here through decays of the heavy triplet higgs, which generates the lepton asymmetry of the universe, which in turn generates a baryon asymmetry of the universe.
\end{abstract}

\footnotetext{
${ }^{1}$ E-mail: utpal@prl.ernet.in

${ }^{2}$ permanent address
} 
Recently the Super Kamiokande [1] announced a positive evidence of neutrino oscillations. They attribute the $\nu_{\mu}$ deficit in the atmospheric neutrino to a $\nu_{\mu}$ oscillating into a $\nu_{a t m}$, where $\nu_{a t m}$ could be a $\nu_{\tau}$ or a sterile neutrinos (which is a singlet under the standard model) with $\Delta m_{\text {atmos }}^{2}=$ $m_{\nu_{\mu}}^{2}-m_{\text {atm }}^{2} \sim(0.5-6) \times 10^{-3} \mathrm{eV}^{2}$. There are also indications of neutrino oscillations in the neutrinos coming from the sun. The solar neutrino deficit can be explained if one considers $\nu_{e} \rightarrow \nu_{\text {sol }}$ oscillations (where $\nu_{\text {sol }}$ could be $\nu_{\mu}$ or $\nu_{\tau}$ or a sterile neutrino) with the mass squared difference [2] $\Delta m_{\text {solar }}^{2}=m_{\nu_{\text {sol }}}^{2}-m_{\nu_{e}}^{2} \sim(0.3-1.2) \times 10^{-5} \mathrm{eV}^{2}$, This mass squared difference is for resonant oscillation [3]. If one assumes vacuum oscillation solution of the solar neutrino deficit, then this number will be several orders of magnitude smaller. If we assume a three generation scenario, $\nu_{a t m}$ is then identified with $\nu_{\tau}$ and $\nu_{\text {sol }}$ could be a $\nu_{\mu}$ or a $\nu_{\tau}$. Consider, $\nu_{\text {sol }} \equiv \nu_{\tau}$, which implies, $m_{\nu_{\mu}}^{2}-m_{\nu_{e}}^{2}=\left[m_{\nu_{\mu}}^{2}-m_{\nu_{\tau}}^{2}\right]+\left[m_{\nu_{\tau}}^{2}-m_{\nu_{e}}^{2}\right]=\Delta m_{\text {atmos }}^{2}+\Delta m_{\text {solar }}^{2} \sim 10^{-2} \mathrm{eV}^{2}$. Then we cannot explain the LSND result [4], which announced a positive evidence of $\overline{\nu_{\mu}} \rightarrow \overline{\nu_{e}}$ oscillations with the mass squared difference (alternate explanation is not possible [5]) $\Delta m_{L S N D}^{2}=m_{\nu_{\mu}}^{2}-m_{\nu_{e}}^{2} \sim(0.2-2) \mathrm{eV}^{2}$. This conclusion is true even if we consider $\nu_{\text {sol }} \equiv \nu_{\mu}$.

As a solution to this problem one can say that either LSND result is wrong or there has to be some other explanation for the solar neutrino deficit. But a more popular solution is to extend the standard model to incorporate a sterile neutrino and explain all these experiments [6]. Since LEP data [7] ruled out any possibility of a fourth $S U(2)_{L}$ doublet left-handed neutrino, this fourth neutrino has to be a sterile neutrino, which does not interact through any of the standard model gauge bosons. Incorporating such light sterile neutrino with large mixing with the other light neutrinos in extensions of the standard model is non-trivial [6]. Recently there is one attempt to extend the radiative neutrino mass generation model by Zee [8] to incorporate a sterile neutrino [9]. However, that model cannot explain the baryon asymmetry of the universe. 
In this article, we propose a new scenario with an $S U(2)$ symmetry, which can provide a naturally light sterile neutrino with large mixing with the other left-handed neutrinos. The neutrino mass matrix with the sterile neutrinos can now explain the LSND data, the solar neutrino problem, the atmospheric neutrino anomaly and the dark matter problem. The lepton number violation at a very high scale generates a lepton asymmetry of the universe, which then gets converted to the baryon asymmetry of the universe during the electroweak phase transition.

We work in an extension of the standard model which includes a couple of heavy triplet higgs scalars [11, 10], whose couplings violate lepton number explicitly at a very high scale, which in turn gives small neutrino masses naturally. Decays of these triplet higgses generates a lepton asymmetry of the universe [10]. We extend this minimal scenario to include a $S U(2)_{S}$ gauge group so as to extend the standard model gauge group to

$$
\mathcal{G}_{\text {ext }} \equiv S U(3)_{c} \times S U(2)_{L} \times U(1)_{Y} \times S U(2)_{S}
$$

The $S U(2)_{S}$ symmetry breaks down alongwith the lepton number at very high energy $(M)$, and the out-of-equilibrium condition for generating baryon asymmetry of the universe determines this scale $M$ [13]. Since all representations of the $S U(2)$ groups are pseudo-real and anomaly free, there is no additional constraints coming from cancellation of anomaly. This makes this mechanism easy to implement in different scenarios.

The fermion and the scalar content of the standard model, which transformations under $S U(3)_{c} \times S U(2)_{L} \times U(1)_{Y}$ as

$$
\begin{array}{rll}
q_{i L} \equiv(3,2,1 / 6) & u_{i R} \equiv(3,1,2 / 3) & d_{i R} \equiv(3,1,-1 / 3) \\
l_{i L} \equiv(1,2,-1 / 2) & e_{i R} \equiv(1,1,-1) & \phi \equiv(1,2,1 / 2)
\end{array}
$$

are all singlets under the group $S U(2)_{S} \cdot i=1,2,3$ is the generation index. The two heavy triplet higgs scalars $\xi_{a} \equiv(1,3,1), a=1,2$, required to give 
masses to the left-handed neutrinos are also singlets under $S U(2)_{S}$. In this mechanism we add a $S U(2)_{S}$ doublet neutral left-handed fermion $S_{L}$ and two scalars $\eta$ and $\chi$, which transform under $\mathcal{G}_{\text {ext }}$ as

$$
S_{L} \equiv(1,1,0,2) \quad \eta \equiv(1,2,1 / 2,2) \quad \chi \equiv(1,1,0,2)
$$

There are two scales in the theory, the $S U(2)_{S}$ and the lepton number violating scale $M$ and the electroweak symmetry breaking scale $m_{W}$. At a high energy $M, \chi$ acquires a vacuum expectation value (vev) and breaks $S U(2)_{S}$. Lepton number is broken explicitly at this scale through the couplings of the scalar triplets. All the new scalars are considered to be very heavy,

$$
M_{\eta} \sim M_{\chi} \sim M_{\xi_{a}} \sim M
$$

The fields $\eta$ and $\xi_{a}$ do not acquire any vev. However, once the standard model higgs doublet $\phi$ acquires a vev, these fields $\xi_{a}$ and $\eta$ acquires a very tiny vev, which in turn gives very small masses and large mixing to the neutrinos.

Consider the most general potential of all the scalars in the model $\left(\xi_{a}, \eta, \chi, \phi\right)$. There will be quadratic and quartic couplings of the form,

$$
M_{H}^{2}\left(H^{\dagger} H\right), \lambda_{H}\left(H^{\dagger} H\right)\left(H^{\dagger} H\right) \text { and } \lambda_{12}^{\prime}\left(H_{1}^{\dagger} H_{1}\right)\left(H_{2}^{\dagger} H_{2}\right)
$$

where $H$ correspond to any one of the scalar fields. In addition, there will be two coupled terms,

$$
\begin{aligned}
V & =\mu_{a}\left(\xi_{a}^{0} \phi^{0} \phi^{0}+\sqrt{2} \xi_{a}^{-} \phi^{+} \phi^{0}+\xi_{a}^{--} \phi^{+} \phi^{+}\right) \\
& +m\left[\phi^{0}\left(\eta_{+}^{0} \chi_{-}-\eta_{-}^{0} \chi_{+}\right)-\phi^{+}\left(\eta_{+}^{-} \chi_{-}-\eta_{-}^{-} \chi_{+}\right)\right] \\
& +m\left[\phi^{0}\left(\eta_{+}^{0} \chi_{-}^{*}-\eta_{-}^{0} \chi_{+}^{*}\right)-\phi^{+}\left(\eta_{+}^{-} \chi_{-}^{*}-\eta_{-}^{-} \chi_{+}^{*}\right)\right]+\text { h.c. }
\end{aligned}
$$

where $\eta_{+}^{-}$represents the component of $\eta$ with electric charge -1 and $T_{3}=$ $+1 / 2$ of $S U(2)_{S} ; \chi_{+}$represents the component of $\chi$ with $T_{3}=+1 / 2$ of $S U(2)_{S}$; and $\chi_{+}^{*}$ is the component of $\chi^{\dagger}$ with $T_{3}=+1 / 2$ of $S U(2)_{S}$. 
For consistency [10] we require $\mu_{a}$ to be less than but of the order of masses of $\xi_{a}$, and we choose

$$
\mu \sim 0.1 M
$$

When the field $\chi$ acquires a vev, a mixing of the fields $\phi$ and $\eta$ of amount $m<\chi>$ will be induced. Since $M_{\eta} \sim M$ and $m_{\phi} \sim m_{W}$, to protect the electroweak scale we then require

$$
m \sim m_{W} .
$$

This fixes all the mass parameters in this scenario. We can now proceed to minimize the potential. In ref [10] it was shown that the triplet higgs scalars get a very small vev consistent with the minimisation of the potential. In the present scenario both the higgs triplet $\xi_{a}$ and the new doublet higgs scalar $\eta$ get a tiny vev on minimisation, without any fine tuning of parameters. We assume that $T_{3}=+1 / 2$ component of $\chi$ acquires a vev. But that can induce vevs to both the neutral $S U(2)_{S}$ components $\eta_{+}^{0}$ and $\eta_{-}^{0}$. They are given by,

$$
\begin{gathered}
<\xi_{a}>\simeq-\frac{\mu<\phi>^{2}}{M_{\xi_{a}}^{2}} \\
<\eta_{-}^{0}>\simeq-\frac{m<\phi><\chi_{+}>}{M_{\eta}^{2}} \text { and }<\eta_{+}^{0}>\simeq-\frac{m<\phi><\chi_{-}^{*}>}{M_{\eta}^{2}} .
\end{gathered}
$$

Since, $\mu \sim M_{\xi_{a}} \sim M_{\eta} \sim<\chi_{+}>\sim M$ and $m \sim m_{\phi} \sim<\phi>\sim m_{w}$, we get,

$$
<\xi_{a}>\sim<\eta_{-}^{0}>\sim<\eta_{+}^{0}>\sim O\left(\frac{m_{W}^{2}}{M}\right) .
$$

The vevs of $\xi_{a}$ now give small masses to the left-handed neutrinos and the vev of $\eta_{ \pm}^{0}$ allows mixing of the $S U(2)_{L}$ singlet neutrinos $S_{L}$ with the usual left-handed neutrinos, both of which are now of the same order of magnitude naturally.

The Yukawa couplings of the leptons are given by,

$$
\mathcal{L}=f_{\alpha i}^{e} \overline{l_{i L}} e_{\alpha R} \phi+f_{a i j} l_{i L} l_{j L} \xi_{a}+h_{i x} \epsilon_{x y} l_{i L} S_{L x} \eta_{y}+\text { h.c. }
$$


where $x, y=1,2$ are the $S U(2)_{S}$ indices. The first term contributes to the charged lepton masses, while the second and third terms contributes to the neutrino mass and mixing matrix. In the basis, $\left[\begin{array}{ll}\nu_{i L} & S_{L x}\end{array}\right]$ we can now write down the mass matrix as,

$$
\mathcal{M}_{\nu}=\left(\begin{array}{cc}
\sum_{a} f_{a i j}<\xi_{a}> & h_{i x} \epsilon_{x y}<\eta_{y}^{0}> \\
h_{i x}^{T} \epsilon_{x y}<\eta_{y}^{0}> & 0
\end{array}\right) .
$$

There is no Majorana mass terms for the sterile neutrinos. We shall now discuss how to generate baryon asymmetry of the universe [12, 13] in this scenario and what constraint it gives on the new mass scale $M$ and then come back to the neutrino mass matrix.

Lepton number is violated when the scalars $\xi_{a}$ decays

$$
\xi_{a} \rightarrow \begin{cases}l_{i}^{c} l_{j}^{c} & (L=-2) \\ \phi \phi & (L=0)\end{cases}
$$

All other couplings conserve lepton number. By assigning a lepton number -1 to $S_{L x}$, we can ensure conservation of lepton number in the decays of the doublet scalar field $\eta$.

We choose the mass matrix of $\xi_{a}$ to be real and diagonal $\left(\begin{array}{cc}M_{\xi_{1}} & 0 \\ 0 & M_{\xi_{2}}\end{array}\right)$; but once the one loop self energy type contributions are included, imaginary phases from $\mu_{a}$ and $f_{a i j}$ makes it complex. The absorptive part of the one loop self-energy type diagram will introduce observable $\mathrm{CP}$ violation in the mass matrix [14], which would produce unequal amount of leptons and antileptons in the decays of the $\xi_{a}^{++}$and $\xi_{a}^{--}$respectively. This will create a charge asymmetry, which will be compensated by equal and opposite amount of charge asymmetry in the production of $\phi^{+}$and $\phi^{-}$in the decays of $\xi_{a}^{++}$ and $\xi_{a}^{--}$, so that the universe remains charge neutral.

The interference of the tree level and the one loop diagram of figure 1 will generate a lepton asymmetry in the decays of $\xi_{a}$, which is given by,

$$
\delta_{a} \simeq \frac{\operatorname{Im}\left[\mu_{1} \mu_{2}^{*} \sum_{k, l} f_{1 k l} f_{2 k l}^{*}\right]}{8 \pi^{2}\left(M_{\xi_{1}}^{2}-M_{\xi_{2}}^{2}\right)}\left[\frac{M_{\xi_{a}}}{\Gamma_{\xi_{a}}}\right] .
$$




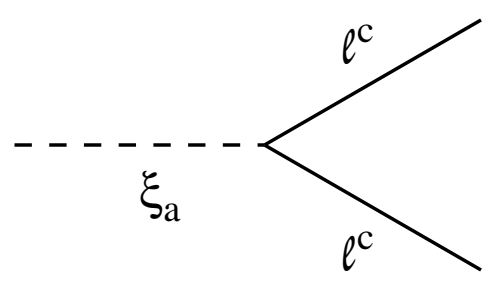

(a)

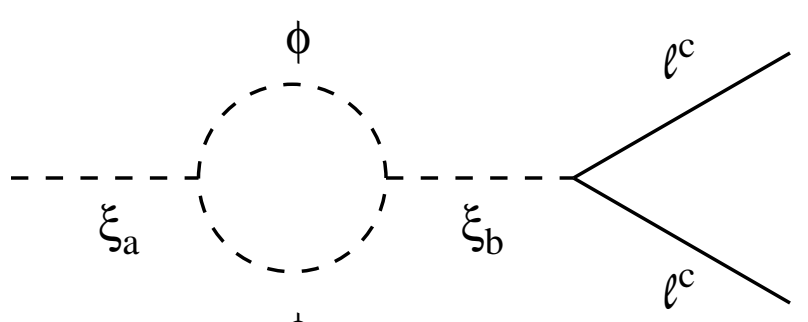

$\phi$

Figure 1: The decay of $\xi_{a} \rightarrow l^{c} l^{c}$ at tree level (a) and in one-loop order (b). $C P$-violation comes from an interference of these diagrams.

where, the decay width of these scalars $\xi_{a}$ is given by,

$$
\Gamma_{\xi_{a}}=\frac{1}{8 \pi}\left(\frac{\left|\mu_{1}\right|^{2}+\left|\mu_{2}\right|^{2}}{M_{\xi_{a}}}+\sum_{i, j}\left|f_{a i j}\right|^{2} M_{a}\right) .
$$

These decays should be slower [13] than the expansion rate of the universe $(H)$, otherwise the lepton asymmetry $\delta_{a}$ in decays of $\xi_{a}$ will be suppressed by an amount $K(\ln K)^{0.6}$, where $K=\frac{\Gamma_{\xi_{a}}}{H} ; H=\sqrt{1.7 g_{*}} \frac{T^{2}}{M_{P l}} \quad$ at $T=M_{\xi_{a}}$; $M_{P l}$ is the Planck scale; and $g_{*}$ is the total number of relativistic degrees of freedom.

We consider $M_{\xi_{2}}<M_{\xi_{1}}$, so that when $\xi_{2}$ decays, $\xi_{1}$ has already decayed away and only the asymmetry $\delta_{2}$ generated in decays of $\xi_{2}$ will contribute to the final lepton asymmetry of the universe. The lepton asymmetry thus generated will be the same as the $(B-L)$ asymmetry of the universe, which will then get converted to a baryon asymmetry during the electroweak phase transition 15. The final baryon asymmetry of the universe is given by,

$$
\frac{n_{B}}{s} \sim \frac{\delta_{2}}{3 g_{*} K(\ln K)^{0.6}} .
$$


To obtain the desired amount of baryon asymmetry of the universe one possibility could be [10], $M_{2}=10^{13} \mathrm{GeV}$, and $\mu_{2}=2 \times 10^{12} \mathrm{GeV}$, which gives us $m_{\nu_{\tau}}=1.2 f_{233} \mathrm{eV}$, assuming that the $M_{1}$ contribution is negligible. Now let $M_{1}=3 \times 10^{13} \mathrm{GeV}, \mu_{1}=10^{13} \mathrm{GeV}$, and $f_{1 k l} \sim 0.1$, then the decay of $\psi_{2}$ generates a lepton asymmetry $\delta_{2}$ of about $8 \times 10^{-4}$ if the $\mathrm{CP}$ phase is maximum. Using $M_{P l} \sim 10^{19} \mathrm{GeV}$ and $g_{*} \sim 10^{2}$, we find $K \sim 2.4 \times 10^{3}$, so that $n_{B} / s \sim 10^{-10}$.

Thus, with the heavy mass scale to be of the order of $M \sim 10^{13-14} \mathrm{GeV}$ it is possible to get the desired amount of baryon asymmetry of the universe and vevs of $\xi$ and $\eta$ to be of the order of a few eV. Then with proper value of the Yukawa couplings $f_{a i j}$ and $h_{i x}$ we can get a neutrino mass matrix (equation 5) which can explain all the neutrino experiments. All the elements of the mass matrix could be non-zero and are about a few eV or less, except for the Majorana mass term of the sterile neutrinos. One can then have several possible scenarios [6] which can explain all the neutrino experiments. Cosider for example [16] one sterile neutrino $\left(S_{L 1}\right)$ with mass of about $(2-3) \times 10^{-3}$ $\mathrm{eV}$, which mixes with the $\nu_{e}$, while the other sterile neutrino $\left(S_{L 2}\right)$ is lighter. This will satisfy the constraints on the sterile neutrinos from nucleosynthesis. If we choose $m_{\nu_{e}}$ to be much lighter than $S_{L 1}$, that satisfies all laboratory constraints on its mass. Then the $\nu_{e} \rightarrow \nu_{S_{1}}$ oscillations can explain the solar neutrino deficit. We can further assume that $\nu_{e} \rightarrow \nu_{S_{1}}$ oscillations satisfies the resonant oscillation condition, while $\nu_{e} \rightarrow \nu_{S_{2}}$ oscillations satisfies the vacuum oscillation condition, so there are both the components.

For a solution of the atmospheric neutrino anomaly we assume the $\left[\begin{array}{ll}\nu_{\mu} & \nu_{\tau}\end{array}\right]$ mass matrix to be of the form,

$$
M=\left(\begin{array}{cc}
m_{a} & m_{a b} \\
m_{a b} & m_{b}
\end{array}\right)
$$

where, $m_{a b}>m_{b}>m_{a}$, so that the two physical states are almost degenerate with masses $m_{a b}$, but the mass squared difference is given approximately by, $m_{\nu_{\mu}}^{2}-m_{\nu_{\tau}}^{2} \sim m_{a b} m_{b}$. We can then have $m_{a b} \sim \mathrm{eV}$ and $m_{b} \sim 10^{-2} \mathrm{eV}$, 
so that the $\nu_{\mu}$ and $\nu_{\tau}$ are almost degenerate with mass about a few $\mathrm{eV}$ to be the hot component of the dark matter and the mass squared difference $m_{\nu_{\mu}}^{2}-m_{\nu_{\tau}}^{2} \sim 10^{-2} \mathrm{eV}^{2}$ and maximal mixing can explain the atmospheric neutrino anomaly. These numbers show the freedom available to the present scenario to explain all the data. In practice, as in the case of quark and charged lepton masses, only future experiments can determine the exact form of the Yukawa couplings $f_{a i j}$ and $h_{i x}$.

To summarise, we propose a simple scenario to accomodate naturally light sterile neutrinos in extensions of the standard model with an $S U(2)_{S}$ symmetry. There are two mass scales in the model, the electroweak scale and the scale of lepton number and $S U(2)_{S}$ violation, which is fixed by the conditions for lepton asymmetry of the universe. The heavy triplet and a doublet acquires very tiny vev, which gives masses and mixing of the lefthanded neutrinos and the sterile neutrinos. The low energy mass matrix can then explain the solar neutrino deficit, atmospheric neutrino anomaly, LSND result and the dark matter problem. The decays of the triplets gener-

ates a lepton asymmetry of the universe, which gets converted to a baryona symmetry of the universe during the electroweak phase transition.

\section{ACKNOWLEDGEMENT}

I would like to thank Prof W. Buchmuller and the Theory Division, DESY, Hamburg for hospitality and acknowledge financial support from the Alexander von Humboldt Foundation. 


\section{References}

[1] T. Kajita, Talk presented at Neutrino 1998 Conference; Y. Fukuda et al, Phys. Lett. B 335, 237 (1994) and references therein.

[2] R. Davis, Prog. Part. Nucl. Phys. 32, 13 (1994); Y. Fukuda et al, Phys. Rev. Lett. 77, 1683 (1996); P. Anselmann et al, Phys. Lett. B 357, 237 (1995); B 361, 235 (1996).

[3] L. Wolfenstein, Phys. Rev. D 17, 2369 (1978); S.P. Mikheyev and A. Yu. Smirnov, Yad. Fiz. 42, 1441 (1995) [Sov. J. Nucl. Phys. 42, 913 (1985)].

[4] A. Athanassopoulos et al (LSND Collaboration), Phys. Rev. Lett. 75, 2650 (1995).

[5] R.B. Mann and U. Sarkar, Phys. Rev. Lett. 76, 865 (1996)

[6] A. Smirnov, report no. hep-ph/9611465.

[7] P.B. Renton, Int. J. Mod. Phys. A12, 4109 (1997).

[8] A. Zee, Phys. Lett. B 93, 389 (1980).

[9] N. Gaur, A. Ghosal, E. Ma and P. Roy, report no. hep-ph/9806272.

[10] E. Ma and U. Sarkar, Phys. Rev. Lett. 80, (1998) 5716.

[11] C. Wetterich, Nucl. Phys. B 187, 343 (1981); G.Lazarides, Q. Shafi and C. Wetterich, Nucl. Phys. B181, 287 (1981); R.N. Mohapatra and G. Senjanovic, Phys. Rev. D 23, 165 (1981); R. Holman, G. Lazarides and Q. Shafi, Phys. Rev. D27, 995 (1983); G. Lazarides and Q. Shafi, report no hep-ph/9803397.

[12] A.D. Sakharov, Pis'ma Zh. Eksp. Teor. Fiz. 5, 32 (1967). 
[13] E.W. Kolb and M.S. Turner, The Early Universe (Addison-Wesley, Reading, MA, 1989).

[14] M. Flanz, E.A. Paschos and U. Sarkar, Phys. Lett. B 345, 248 (1995); M. Flanz, E.A. Paschos, U. Sarkar and J. Weiss, Phys. Lett. B 389, 693 (1996).

[15] M. Fukugita and T. Yanagida, Phys. Lett. B 174, 45 (1986); J. A. Harvey and M. S. Turner, Phys. Rev. D42, 3344 (1990).

[16] D.O. Caldwell and R.N. Mohapatra, Phys. Rev. D 48, 3259 (1993); J. Peltoniemi and J.W.F. Valle, Nucl. Phys. B406, 409 (1993). 